\title{
Ablative Treatment of Hepatic Recurrence of Lung Cancer Using Electrochemotherapy : A Case Report
}

\author{
Giuseppina Pacella, Eliodoro Faiella, Carlo Altomare, Bruno Beomonte Zobel, and Rosario Francesco Grasso* \\ Department of Radiology, University of Rome "Campus Bio-medico", Via Alvaro del Portillo, 21- 00128 Rome, Italy
}

${ }^{\star}$ Corresponding author: Rosario Francesco Grasso, PhD MD, Department of Radiology, Campus Bio-medico University of Rome, Via Alvaro del Portillo, 21- 00128 Roma , Italy; Tel: +3906.225411669; Email: r.grasso@unicampus.it

Received: May 29, 2019; Accepted: June 13, 2019; Published: June 15, 2019

\begin{abstract}
Introduction: Electrochemotherapy (ECT) is a locally enhanced chemotherapy that combines the administration of chemotherapeutic drugs with welldosed electric pulses for cell membrane Electroporation (EP). As opposed to thermal ablation, cell death with ECT is primarily induced using electrical energy: electrical pulses disrupt the cellular membrane integrity, resulting in cell death while sparing the extracellular matrix of sensitive structures such as the bile ducts, blood vessels, and bowel wall. This article reports the successful non-thermal ablation treatment of a hepatic recurrence of lung cancer as an individual treatment in order to achieve loco-regional tumor control.
\end{abstract}

Case Presentation: 50-year-old Caucasian woman was referred for interventional treatment of the largest of the hepatic metastases of lung cancer (6.0 $\times$ $4.8 \mathrm{~cm}$ ), located in the left hepatic lobe and close to the left suprahepatic vein. Due to the size and the immediate proximity to the left suprahepatic vein the patient could neither undergo ablation treatments (RFA or MWA) neither TACE because of tumor size and the high risk of thermal sinks ("heat-sink effect") potentially resulting in reduction of complete treatment of the target lesion. Due to its ablation mechanism, electrochemotherapy with use of bleomicin was deemed to be the best therapy option for the patient as loco-regional disease control.

Conclusions: Due to its more selective and non-thermal ablation effect, percutaneous ECT is a novel, potentially very effective treatment option in minimally invasive oncologic treatments, especially for hepatic metastases. We showed in this case report that a large hepatic metastatic lesion adjacent to the left suprahepatic vein can be widely ablated by ECT with ablation of a large infiltrating tissue volume.

Keywords: Electrochemotherapy, Reversible Electroporation, Hepatic Metastases.

\section{Introduction}

Electrochemotherapy (ECT) is a locally enhanced chemotherapy that combines the administration of chemotherapeutic drugs with well-dosed electric pulses for cell membrane electroporation (EP) [1].

Tissue electroporation is a novel approach to introduce molecules and genes into the cells of specific areas of the body. It employs the ability of certain electrical fields to reversibly permeabilize the cell membrane in a process known as reversible electroporation [2].

The exposure of biological membranes to a sufficiently high external electric field can lead to a rapid and large increase in electric conductivity and permeability, called membrane EP. While applying an electric field on cell membranes, their surface tension will be destabilized and nonpermanent molecules can diffuse into the cytosol. According to the theory of aqueous pore formation, pores are able to form spontaneously, when the bilayer is exposed to an electrical field $(>50 \mathrm{~V})[2,3]$.

Mass transfer can now occur through these channels (pores), which in reversible electroporation persist for a period of a few seconds. Typically, in reversible electroporation, the permeabilization pulses are delivered on a time scale of microseconds, and the pores reseal on a time scale of seconds. Macromolecules in the extracellular space can enter the cells by diffusion during the time the pores are open [4].

When electric pulses are applied to cells, 2 different phenomena are observed: reversible EP and irreversible electroporation (IRE), both used in clinical practice. Reversible EP will increase cell membrane permeability and open an access route for molecules that are too big to cross the cell membrane (DNA, RNA) or facilitates cell enter by hydrophilic molecules (bleomycin [BLM], cisplatin) [5-7]. These molecules once crossed the cell membrane exert their effect in the resealing and intact cells. In contrast, IRE $(>600 \mathrm{~V} / \mathrm{cm})$ is used as non-thermal form of soft tissue ablation in clinical routine [8]. The IRE of cell membranes leads to a disturbance of the cell homeostasis and thus ultimately to apoptosis in the treated tissue [9-11].

Electrochemotherapy is a promising method to locally treat tumors regardless of its histological type with minimal adverse side effects and a high response rate $[12,13]$.

The efficacy of ECT treatment is well demonstrated for cutaneous and subcutaneous melanomas, and the technique can be applied in a variety of malignant lesions $[14,15]$. The range of applications can be divided into 3 groups: (1) treatment of cutaneous and subcutaneous metastases located in head or neck, melanoma, non-melanoma 
skin cancer, or breast cancer metastases to the skin [14, 16-19]; (2) treatment of non-cutaneous metastases located in bone, liver, or Soft Tissue Sarcoma (STS) [20-22]; and (3) clinical trials for the treatment of primary tumors, such as ovary or colon cancer [23,24].

Its applications were described by Neumann et al nearly 3 decades ago when electrical fields were used to temporarily create pores in cell membrane to facilitate gene transfer into mouse lyoma cells [25]. The use of electroporation to increase the permeability of the cell membrane in tissue was introduced by Okino and Mohri in 1987 [26] and by Mir et al in 1991 [27], who described that combining an impermeant anticancer drug with reversibly permeabilizing electrical pulses greatly enhanced the effectiveness of the treatment compared with either therapy alone.

The liver is an organ of particular current research interests in ECT. Besides established ablation modalities like microwave ablation [28] or RFA [29] and IRE [30] there is need for a controlled non-thermal ablation modality that enhances the efficacy of chemotherapeutic agents. The benefit of non-thermal ablation, like IRE, is the ability to treat lesions without thermonecrosis. This enables the ability to ablate near-sensitive structures like vessels and nerves, as no heat will be produced which spread around the treated area. An already established loco-regional method is TACE. During TACE, embolizing agents and chemotherapeutics will be administrated via catheter to obtain tissue-specific necrosis. As both therapy concepts are based on loco-regional application of cytostatic in hepatocellular parenchyma, a similar response rate of ECT by liver lesions can be possible. Similar to TACE, ECT allows for a controlled loco-regional additional chemotherapy without marked systemic side effects. As the chemotherapeutics applied during ECT are membrane impermeant, even better results might be possible [1].

First trials of ECT by hepatic tumors were conducted in animal models. Electrochemotherapy was shown to be effective to reduce the volume of hepatic metastases of colorectal cancer in the rats [31].

Electrochemotherapy of solid organs has been evaluated in phase I and II trials in humans [21, 32]. A significant reduction of viable tumor tissue in ECT-treated metastases was observed. Infarct-like necrosis occurred presumably caused by the cytotoxic and vasculardisrupting effect on tumor cells and small tumor blood vessels $[32,33]$.

Histopathological analysis of colorectal liver metastases after ECT treatment revealed necrotic and fibrotic changes of tumor and normal tissue in the treated area, whereas 3 months later, regeneration was observable. The analysis also revealed that after ECT treatment, most vessels ( $>5 \mathrm{~mm}$ ) and biliary structures were preserved [34].

In a small clinical trial, patients received an open approach of ECT for the treatment of unresectable colorectal liver metastases. The obtained response rates 4 weeks after ECT were $55 \%$ as complete response and $45 \%$ stable disease [35].

A recent study by Gasljevic et al. [34] Demonstrated regressive changes in the whole ECT-treated area of the liver. It confirmed that ECT could be proposed for the therapy of metastases near major blood vessels in the liver to provide a safe approach with good antitumor efficacy.

The successful establishment of ECT as hepatic lesion treatment can offer an additional minimally invasive treatment in the case of malignant lesions with decreased systemic side effects.

This article reports the successful non-thermal ablation treatment of a hepatic recurrence of lung cancer as an individual treatment in order to achieve loco-regional tumor control.

\section{Case Presentation}

A 50-year-old Caucasian woman was referred for treatment of one of the liver metastases of lung cancer. After initial diagnosis of lung cancer with multiple bone metastases and a single hepatic lesion in 2013, a systemic chemiotherapy was performed with subsequent radiotherapy with the initial result of partial tumor remission. The hepatic lesion was treated with microwave ablation and was obtained a great loco-regional tumor control.

Under systemic chemotherapy (denosumab and navelbine), after a partial remission and subsequent disease stability, in 2018 a systemic progression was observed with appearance of peritoneal carcinomatosis, multiple lymphadenopathy, brain metastases and numerical and dimensional increase of bone and hepatic metastases.

The largest of the hepatic lesions was located in the left hepatic lobe and close to the left suprahepatic vein. The tumor size was $6.0 \times$ $4.8 \mathrm{~cm}$ in axial section at the abdominal CT scan (Figure 1).

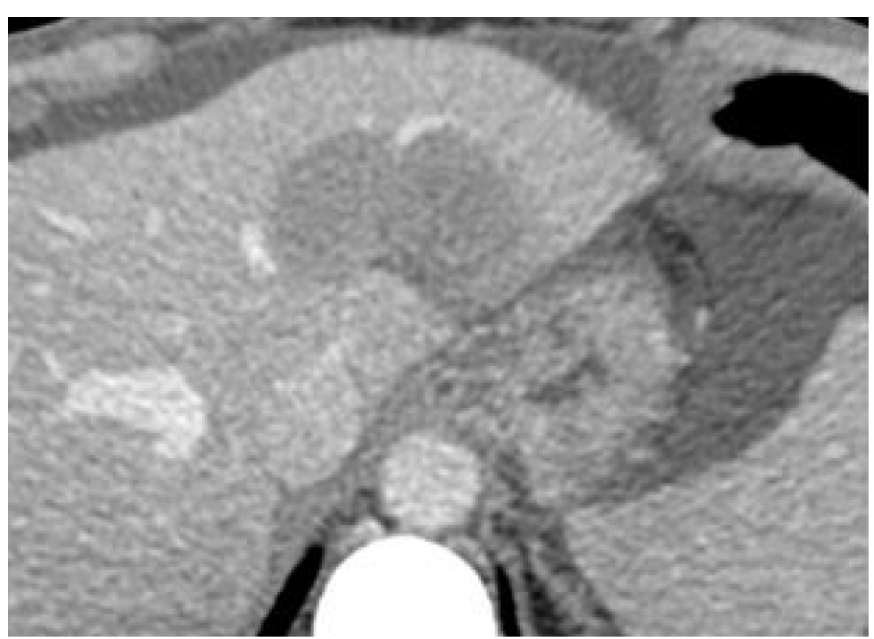

Figure 1. Contrast-enhanced axial CT scan demonstrating the large hepatic metastases $(6.0 \times 4.8 \mathrm{~cm})$ located in the left hepatic lobe and close to the left suprahepatic vein prior to ECT procedure. CT indicates computed tomography; ECT indicates electrochemotherapy.

The patient's case was discussed at the multidisciplinary tumor board for therapy options: due to the size and the immediate proximity to the left suprahepatic vein the patient could neither second ablation treatments (RFA or MWA) nor TACE because of tumor size and the high risk of thermal sinks ("heat-sink effect") potentially resulting in reduction of complete treatment of the target lesion. Electrochemotherapy with use of bleomicin was deemed to be the best therapy option for the patient as loco-regional disease control. 
The concept of ECT is based on the aforementioned properties of reversible EP combined with therapeutic efficacy of chemotherapeutic agents. Due to the increased permeability of the cell membrane, chemotherapeutic agents can pass into cells and induce cell death mitosis in the targeted tissue. Electrodes located around or inside the tumor will deliver defined electric pulses which enables diffusion of otherwise membrane nonpermeant anticancer drug into the target cells [1]. This - in contrast to thermal ablation techniques like Radiofrequency Ablation (RFA) or Microwave Ablation (MWA) potentially allows tumor cell ablation without concomitant destruction of connective tissue, blood vessels and nerves.

Due to this potentially selective cell ablation technique, ECT was offered as a therapy option because it provided the opportunity of tumor mass reduction and decrease of tumor burden with reduced risk of impairment of surrounding blood vessels. The procedure with risks and benefits was discussed with the patient and informed consent was obtained.

The patient was put under general anesthesia and neuromuscular blocking to prevent arrhythmia. The procedure was performed using a commercially available RE system. Due to the large tumor volume a total of five needles were placed into the target area (Figure 2). The percutaneous placement of the electrodes was guided by ultrasound using a multifrequency probe ( 1 to $5 \mathrm{MHz}$ ). As recommended by the manufacturer and recorded by the RE generator the following parameters were used: number of electrodes: five; type of electrodes: monopolar; distance of electrodes: $0.5 \mathrm{~m}$ (minimum), $1.5 \mathrm{~cm}$ (maximum); impulses per electrode: 80 ; voltage: $500 \mathrm{~V}$ (minimum), 3000V (maximum); maximum deliverable current: 50A. An intravenous administration of an anticancer drug (BLM) was done so it could evenly distribute over the vascular system and extracellular space of the tissue. After that, electrodes located around or inside generated the electric pulses for a time of 8 minutes with consequent diffusion of otherwise membrane nonpermeant anticancer drug into the target cells. After a short time, a few seconds to several minutes after exposure to the electric field, the membrane permeability would return to its initial state and the specific chemotherapeutics would cause multiple DNA breaks (BLM) in the abnormal tumor cells. A stepwise ablation procedure with replacement of two electrodes at the level of the caudal portion of the lesion was performed.

During the electrochemotherapy the patient did not have any cardiovascular events, in particular no supraventricular tachycardia and no atrial fibrillation. Complications, especially post-interventional bleeding, were not observed.

Follow-up imaging, after one week, showed good response to the treatment of the hepatic lesion in absence of remaining viable tumor tissue. Contrast-enhanced CT scan at 3 and 6 months after electrochemotherapy procedure showed a complete necrosis of the tumor and reduction of tumor volume (Figure 3).

\section{Discussion}

The majority of patients who are diagnosed with liver malignancies are not eligible for resection or transplantation due to inadequate functional liver function, multifocal or advanced disease, prohibitive tumor location or the presence of medical co-morbidities. In some highly selective scenarios resection appears to remain superior in terms of disease recurrence rates and overall and disease-free survival. For curative intentions in term of local tumor control there are reports of equivalent results following local-regional ablation treatments when compared to resection in selected patients [36-38].

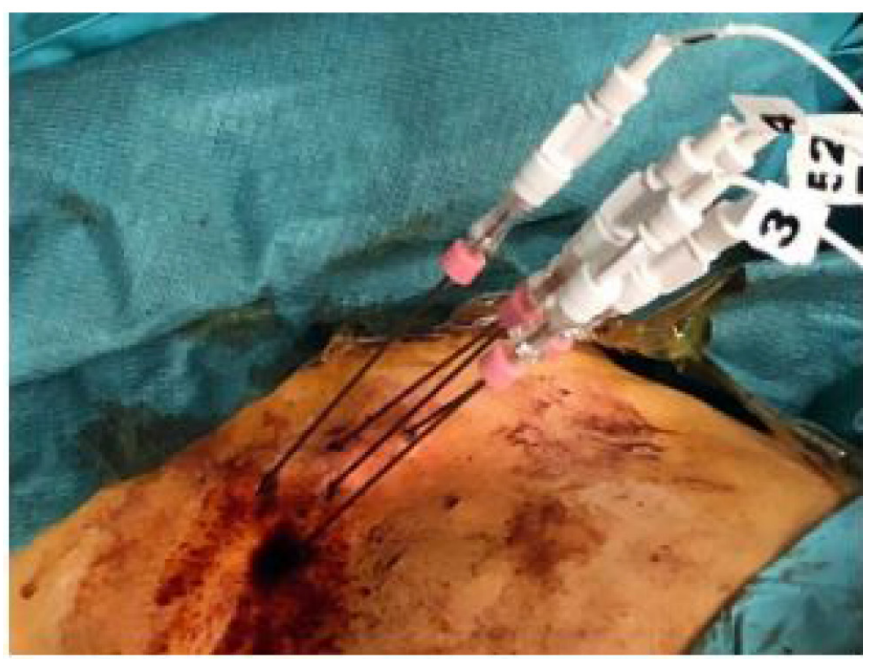

Figure 2. Percutaneous placement of five electrode needles for hepatic lesion treatment.

Image-guided tumor ablation techniques have significantly broadened the treatment possibilities for primary and secondary hepatic malignancies. A tumor resection leads to immediate absence of tumor tissue, in contrast (thermo)ablative procedures will induce necrotic damages whereas chemoablative procedures induce cell apoptosis [1].

Monopolar RFA is an established technique for the treatment of tumors that are limited in number ( 3 or less) and size ( $3 \mathrm{~cm}$ or less) and are located $1 \mathrm{~cm}$ or more from critical structures and vessels. MWA appears to have the potential to improve the rate of complete ablation achieved with RFA in tumors that are larger than $3 \mathrm{~cm}$ or when multiple and seems to have the potential to overcome the limitations of RFA in the treatment of tumors in perivascular locations [39].

A new ablation technique, Reversible Electroporation (RE) with use of chemiotherapy (as BLM), is recently added to the treatment armamentarium. As opposed to thermal ablation, cell death with ECT is primarily induced using electrical energy: electrical pulses disrupt the cellular membrane integrity, resulting in cell death while sparing the extracellular matrix of sensitive structures such as the bile ducts, blood vessels, and bowel wall. The preservation of these structures makes electrochemotherapy attractive for liver metastases that are unsuitable for resection and thermal ablation owing to their anatomical location. In contrast to chemoembolization techniques, ECT relies on membrane non-permeant chemotherapeutics, which need EP for cell uptake. Improvements need to be done to achieve homogeneous EP in the treated area, as well as steady concentrations of cytostatic drug in big lesions [1].

The establishment and expansion of ECT in deep-seated tumors (eg, liver, bone metastases) will open up new opportunities for minimally 
invasive treatment of metastases and carcinomas. Even if only few experiences have been published for colorectal liver metastases treated by ECT, considering the proven safety and the promising results, this treatment option deserves further attention. ECT can induce tumor volume shrinkage, suggesting an implementation in clinical routine as neoadjuvant treatment to enhance future tumor resection. In most cases, it is used in treatment of advanced neoplastic lesions in which radical surgical treatment is not possible (eg, due to lesion location, size, and/or number) [1].
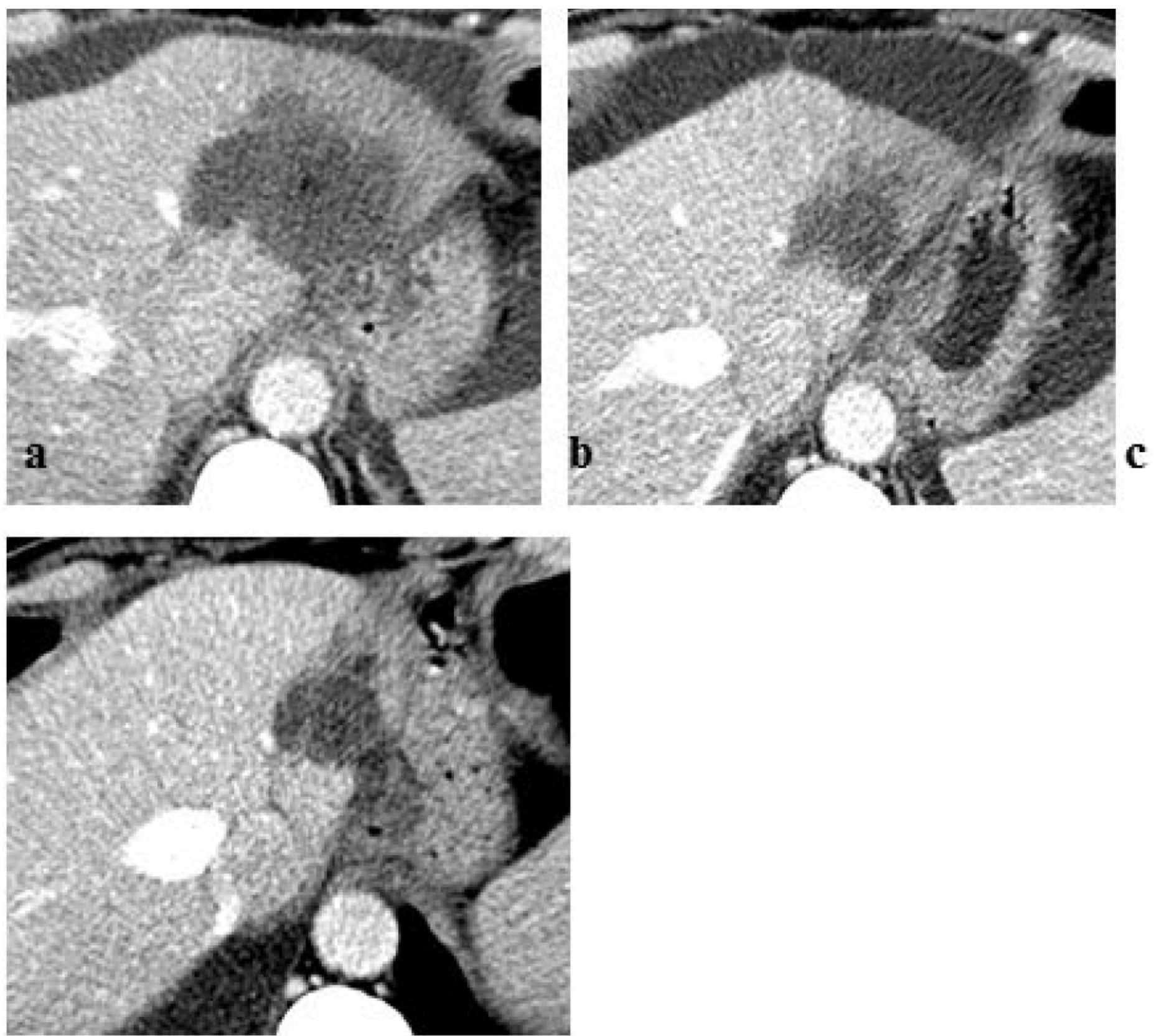

Figure 3. Follow-up imaging post-ECT procedure: contrast-enhanced axial CT scan (a) at one week demonstrating no residual or recurrent tumor; (b) at 3 and (c) 6 months showing a complete necrosis of the tumor and reduction of tumor volume. CT indicates computed tomography; ECT indicates electro chemotherapy.

Even though percutaneous ablation techniques are used as possibly curative therapies, palliative tumor ablation can be useful to achieve loco-regional control of tumor growth, pain relief or pain control, especially in patients with unresectable tumor manifestations [40]. Indeed, electrochemotherapy is usually applied in palliative settings for patients with unresectable tumors, resulting in amelioration of quality of life.

Electrochemotherapy allows treating tumor nodules in the proximity of important structures like vessels and nerves and the safety profile of ECT is favorable. Due to heat dissipation to adjacent 
structures there is an inherent risk of thermal damage of adjacent organs, blood vessels and nerves. Thus, lesions close to adjacent structures with high risk of unintended heat destruction still pose a challenge for percutaneous thermal ablation techniques [41]. In particular, ECT - in contrast to thermal ablation - would allow tumor cell ablation without concomitant destruction of connective tissue, blood vessels and nerves, which means ablation of tumor cells in those areas where thermal ablation was not possible before. In the proximity of larger blood vessels thermal ablation techniques are also hindered by the heat-sink effect. Due to its cooling effect blood flow is an important determinant as much as a limiting factor of thermal ablation techniques $[42,43]$. ECT seems to be unaffected by the blood flow and conversely does not potentially affect the macro vascularization of the ablation zone.

Most of the observed adverse events are local and transient, including moderate local pain, erythema, edema, and muscle contractions during ECT. No serious adverse events or deaths related to ECT have been reported. Limitations of ECT are the need for interventional individual electric pulse generating systems, individual electrode needles, and complex preinterventional planning. As the success of difficult interventions in deep-seated tumors will rely on accurate needle placement, robotic navigated systems as well as image guidance improve successful ECT treatment and minimize reintervention [1].

\section{Conclusion}

Percutaneous ECT of solid organs is a novel, potentially very effective treatment option in minimally invasive oncologic treatments, especially for hepatic metastases.

Due to its more selective and non-thermal ablation effect ECT widens the field of minimally invasive treatable lesions. We showed in this case report that a large hepatic metastatic lesion adjacent to the left suprahepatic vein can be widely ablated by ECT with ablation of a large infiltrating tissue volume.

\section{References}

1. Probst U, Fuhrmann I, Beyer L et al (2018) Electrochemotherapy as a New Modality in Interventional Oncology: A Review. Technol Cancer Res Treat 17:1533033818785329.

2. Weaver J, Chizmadzhev Y (1996) Theory of electroporation: a review. Bioelectrochem Bioenerg 41: 135-160.

3. Pliquett U, Langer R, Weaver J (1995) Changes in the passive electrical properties of human stratum corneum due to electroporation. Biochim Biophys Acta 1239: 111-121.

4. Yair Granot and Boris Rubinsky (2008) Mass Transfer Model for Drug Delivery in Tissue Cells with Reversible Electroporation. Int J Heat Mass Transf 51: 56105616.

5. Belehradek M, Domenge C, Luboinski B, et al. (1993) Electrochemotherapy, a new antitumor treatment. First clinical phase I-II trial. Cancer 72: 3694-3700.

6. Calvet C, Andre F, Mir L. (2014) Dual therapeutic benefit of electroporationmediated DNA vaccination in vivo. Oncoimmunology 3: 28540.

7. Ivanov MA, Lamrihi B, Szyf M, et al. (2003) Enhanced antitumor activity of a combination of MBD2-antisense electrotransfer gene therapy and bleomycin electrochemotherapy. J Gene Med 5: 893-899.

8. Onik G, Mikus P, Rubinsky B (2007) Irreversible electroporation: implications for prostate ablation. Technol Cancer Res Treat 6: 295-300.

9. Miller L, Leor J, Rubinsky B (2005) Cancer cells ablation with irreversible electroporation. Technol Cancer Res Treat 4: 699-705.
10. Wiggermann P, Bruenn K, Baeumler W (2017) Irreversible electroporation (IRE): a minimally invasive therapeutic option in prostate cancer [in German]. Radiologe 57: 637-640

11. Niessen C, Thumann S, Beyer L, et al. (2017) Percutaneous irreversible electroporation: long-term survival analysis of 71 patients with inoperable malignant hepatic tumors. Sci Rep 7: 43687.

12. Jaroszeski MJ, Gilbert R, Nicolau C, et al. (1999) In vivo gene delivery by electroporation. Advanced Drug Delivery Reviews 35: 131-137.

13. Dev SB, Rabussay DP, Widera G, et al. (2000) Medical Applications of Electroporation. IEEE Transactions on Plasma Science 28: 206-223.

14. Marty M, Sersa G, Garbay J, et al. (2006) Electrochemotherapy - an easy, highly effective and safe treatment of cutaneous and subcutaneous metastases: results of ESOPE (European Standard Operating Procedures of Electrochemotherapy) study. EJC Suppl 4: 3-13.

15. Mali B, Jarm T, Snoj M, etal. (2013)Antitumor effectiveness of electrochemotherapy: a systematic review and metaanalysis. Eur J Surg Oncol 39: 4-16.

16. Quaglino P, Mortera C, Osella-Abate S, et al. (2008) Electrochemotherapy with intravenous bleomycin in the local treatment of skin melanoma metastases. Ann Surg Oncol 15: 2215-2222.

17. Eggermont A (1996) Treatment of melanoma in-transit metastases confined to the limb. Cancer Surv 26: 335-349.

18. Sersa G, Cufer T, Cemazar M, et al. (2000) Electrochemotherapy with bleomycin in the treatment of hypernephroma metastasis: case report and literature review. Tumori 86: 163-165.

19. Campana L, Mocellin S, Basso M, et al ( 2009) Bleomycin-based electrochemotherapy: clinical outcome from a single institution's experience with 52 patients. Ann Surg Oncol 16: 191-199.

20. Fini M, Salamanna F, Parrilli A, et al. (2013) Electrochemotherapy is effective in the treatment of rat bone metastases. Clin Exp Metas 30: 1033-1045.

21. Edhemovic I, Gadzijev E, Brecelj E, et al. (2011) Electrochemotherapy: a new technological approach in treatment of metastases in the liver. Technol Cancer Res Treat 10: 475-485.

22. Linnert M, Iversen H, Gehl J (2012) Multiple brain metastases-current management and perspectives for treatment with electrochemotherapy. Radiol Oncol 46: 271-278.

23. Perrone A, Cima S, Pozzati F, et al. (2015) Palliative electrochemotherapy in elderly patients with vulvar cancer: a phase II trial. J Surg Oncol 112: 529-532.

24. Pellegrino A, Damiani G, Mangioni C, et al. (2016) Outcomes of bleomycin-based electrochemotherapy in patients with repeated loco-regional recurrences of vulvar cancer. Acta Oncol 55: 619-624.

25. Neumann E, Schaefer-Ridder M, Wang Y, et al. (1982) Gene transfer into mouse lyoma cells by electroporation in high electric fields. EMBO J 1:841-845

26. Okino M, Mohri H (1987) Effects of a high-voltage electrical impulse and an anticancer drug on in vivo growing tumors. Jpn J Cancer Res 78: 1319-1321

27. Mir LM, Orlowski S, Belehradek J Jr, et al. (1991) Electrochemotherapy potentiation of antitumour effect of bleomycin by local electric pulses. Eur J Cancer 27: 68-72.

28. Simon C, Dupuy D, Mayo-Smith W (2005) Microwave ablation: principles and applications. Radiographics 25: 69-83.

29. Gazelle G, Goldberg S, Solbiati L, et al. (2000) Tumor ablation with radiofrequency energy. Radiology 217: 633-646.

30. Rubinsky B, Onik G, Mikus P (2007) Irreversible electroporation: a new ablation modality - clinical implications. Technol Cancer Res Treat 6: 37-48.

31. Chazal M, Benchimol D, Baque P, et al. (1998) Treatment of hepatic metastases of colorectal cancer by electrochemotherapy: an experimental study in the rat. Surgery 124: 536-540.

32. Edhemovic I, Brecelj E, Gasljevic G, et al. (2014) Intraoperative electrochemotherapy of colorectal liver metastases. J Surg Oncol 110: 320-327

33. Sersa G, Jarm T, Kotnik T, et al. (2008) Vascular disrupting action of electroporation and electrochemotherapy with bleomycin in murine sarcoma. Br J Cancer 98: 388-398.

34. Gasljevic G, Edhemovic I, Cemazar M, et al. (2017) Histopathological findings in colorectal liver metastases after electrochemotherapy. PLos One 12: 0180709.

35. Coletti L, Battaglia V, De Simone P, et al. (2017) Safety and feasibility of electrochemotherapy in patients with unresectable colorectal liver metastases: a pilot study. Int J Surg 44: 26-32.

36. American Association for the Study of Liver Diseases - Practice Guidelines.

37. Diaz-Nieto R, Fenwick S, Malik H, et al. (2017) Defining the Optimal Use of Ablation for Metastatic Colorectal Cancer to the Liver Without High-Level Evidence. Curr Treat Options Oncol 18: 8.

38. Alice Gillams, Nahum Goldberg, Muneeb Ahmed, et al. (2015) Thermal ablation of colorectal liver metastases: a position paper by an international panel of ablation experts, the interventional oncology sans frontières meeting 2013. Eur Radiol 25: $3438-3454$. 
39. Riccardo Lencioni, Thierry de Baere, Robert C. Martin, et al. (2015) Image-Guided Ablation of Malignant Liver Tumors: Recommendations for Clinical Validation of Novel Thermal and Non-Thermal Technologies - A Western Perspective Liver Cancer. 4: 208-214.

40. Mylona S, Karagiannis G, Patsoura S, et al. (2012) Palliative treatment of rectal carcinoma recurrence using radiofrequency ablation. Cardiovasc Intervent Radiol 35: $875-882$.

41. Christoph Niessen, Ernst-Michael Jung, Andreas G Schreyer, et al. (2013) Palliative treatment of presacral recurrence of endometrial cancer using irreversible electroporation: a case report. Journal of Medical Case Reports 7:128
42. Patterson EJ, Scudamore CH, Owen DA, et al. (1998) Radiofrequency ablation of porcine liver in vivo: effects of blood flow and treatment time on lesion size. Ann Surg 227: 559-565.

43. Charpentier KP (2012) Irreversible electroporation for the ablation of liver tumors: are we there yet? Arch Surg - Chicago 147: 1053-1061.

Citation:

Pacella G, Faiella E, Altomare C, Zobel BB, Grasso RF (2019) Ablative Treatment of Hepatic Recurrence of Lung Cancer Using Electrochemotherapy : A Case Report. Interv Med Clin Imaging Volume 2(1): 1-6. 